\title{
Feynman's Path Integrals and Bohm's Particle Paths
}

\author{
Roderich Tumulka*
}

February 18, 2005

\begin{abstract}
Both Bohmian mechanics, a version of quantum mechanics with trajectories, and Feynman's path integral formalism have something to do with particle paths in space and time. The question thus arises how the two ideas relate to each other. In short, the answer is, path integrals provide a re-formulation of Schrödinger's equation, which is half of the defining equations of Bohmian mechanics. I try to give a clear and concise description of the various aspects of the situation.
\end{abstract}

PACS. 03.65.Ta. Key words: Bohmian mechanics, Feynman path integrals, particle trajectories in quantum physics

\section{Background}

Bohmian mechanics [1, 2, 3, 4, 5], invented by D. Bohm in 1952, is a (nonrelativistic) quantum theory without observers; in a world governed by this theory, quantum particles have precise trajectories, and observers find the statistics of outcomes of their experiments in agreement with quantum mechanics. For a system of $N$ particles, their positions $Q_{i}(t) \in \mathbb{R}^{3}$ change according to the equation of motion,

$$
\frac{d Q_{i}(t)}{d t}=\frac{\hbar}{m_{i}} \operatorname{Im} \frac{\psi_{t}^{*} \nabla_{i} \psi_{t}}{\psi_{t}^{*} \psi_{t}}\left(Q_{1}(t), \ldots, Q_{N}(t)\right) .
$$

Here, $m_{i}$ is the mass of particle $i, \phi^{*} \psi$ denotes the scalar product in $\mathbb{C}^{k}$, and $\psi_{t}$ : $\mathbb{R}^{3 N} \rightarrow \mathbb{C}^{k}$ is the wave function of non-relativistic quantum mechanics, defined on the configuration space and evolving according to the (nonrelativistic) Schrödinger equation. In a typical Bohmian universe, the positions $Q_{i}(t)$ appear random, at any time $t$, with joint distribution $\left|\psi_{t}\right|^{2}[3,4]$.

The method of path integrals [6, 7, 8, invented by R. P. Feynman in 1942, is nowadays widely used in quantum physics. I briefly recall the key idea. Let us

*Dipartimento di Fisica dell'Università di Genova and INFN sezione di Genova, Via Dodecaneso 33, 16146 Genova, Italy. E-mail: tumulka@mathematik.uni-muenchen.de 
assume for simplicity a finite set $\mathcal{Q}$ as configuration space (just as a mathematical model), and that time is discrete, too. Then the time evolution of the wave function $\psi$ is defined by a unitary operator $U$ representing one time step,

$$
\psi_{t+1}(r)=\sum_{q \in \mathcal{Q}} U(r, q) \psi_{t}(q)
$$

where $q$ and $r$ run through all configurations, and $U(r, q)$ are the matrix elements of $U$ in the position representation. It follows that after $s$ time steps,

$$
\psi_{t+s}(r)=\sum_{q_{t}, \ldots, q_{t+s-1} \in \mathcal{Q}} U\left(r, q_{t+s-1}\right) \cdots U\left(q_{t+2}, q_{t+1}\right) U\left(q_{t+1}, q_{t}\right) \psi_{t}\left(q_{t}\right)
$$

Since the sum is over all paths, what we have is a path integral! What I want to point out with this little calculation is that path integrals are a mathematical formulation of the time evolution of $\psi$, an equivalent alternative to the Schrödinger equation. The more general formula, for continuous time and configuration space $\mathcal{Q}$, reads

$$
\psi_{t+s}(r)=\int D \gamma e^{i S[\gamma] / \hbar} \psi_{t}\left(\gamma_{t}\right)
$$

where the integration is over all paths $\gamma$ in $\mathcal{Q}$ during the time interval $[t, t+s]$ with $\gamma_{t+s}=r$, and $S[\gamma]$ denotes the (classical) action of the path $\gamma$.

\section{Discussion}

Since the same $\psi$ as in usual quantum mechanics appears in Bohmian mechanics, and with the same time evolution, path integrals are equally relevant to Bohmian mechanics as to usual quantum mechanics. Whatever can be calculated about the evolution by means of path integrals in ordinary quantum mechanics is equally correct in Bohmian mechanics. Note that the path integrals concern only the wave function, not the Bohmian paths. It may be helpful at this point to keep in mind that path integrals are not exclusive to quantum theory: one can just as well re-write Maxwell's equations of classical electrodynamics, as in fact any linear field equation, in terms of path integrals.

An obvious but basic fact that I want to emphasize is that the paths of the path integral have a very different status from the Bohmian paths: Feynman's paths are mathematical tools for computing the evolution of $\psi$, while one among Bohm's paths is the actual motion of the Bohmian particle, which exists in addition to $\psi$.

Now there is another, completely different relation between path integrals and theories of actual particle trajectories: since one considers a lot of possible paths of the particle, is it perhaps one of these paths that the particle actually follows? If the path integral formalism provided a probability distribution on the space of all paths, one could assume that nature chooses one of the paths at random according to this distribution. This theory would have the same ontology as Bohmian mechanics, but a different, in fact stochastic, law of motion. That would seem like a completely 
reasonable and acceptable theory, and if it predicted correctly all probabilities for measurements, as prescribed by the quantum formalism, it would be a serious candidate for the explanation of how the quantum world really works.

But do we have a probability measure on path space? One of the central objects in the path integral formalism is a complex measure $D \gamma e^{i S[\gamma] / \hbar}$ on path space (though it is not rigorously defined in the continuum case, see the last paragraph below). To obtain a real measure, we could take, for example, its positive real or its positive imaginary part or its modulus, but the probabilities for the outcomes of measurements entailed by that measure will not agree with quantum mechanics (in contrast to those in Bohmian mechanics). A better probability measure on path space is not in sight.

Thus, the formulation "possible paths of the particle" for the paths considered in a path integral, a formulation that comes to mind rather naturally, cannot, in fact, be taken literally. The status of the paths is more like "possible paths along which a part of the wave may travel," to the extent that waves travel along paths. For example, in the double-slit experiment some paths pass through one slit, some through the other; correspondingly, part of the wave passes through one slit and part through the other.

(In brackets, a few more remarks on measures on path space. When one inserts imaginary numbers for the time variable, the complex measure $D \gamma e^{i S[\gamma] / \hbar}$ on path space turns into a probability measure defining a stochastic process [8]; the corresponding path integrals are known as Feynman-Kac integrals and useful for computing the operators $e^{-\beta H}$ instead of $e^{-(i / \hbar) t H}$. But the process does not, of course, provide us with a quantum theory without observers, as physical time is not imaginary.)

Finally, I address mathematical rigor. There is a mathematical problem with path integrals when both the time axis and configuration space are taken to be a continuum, a problem that is absent when either one is taken to be a discrete set. The problem is that there exists no analogue to Lebesgue measure, in other words no natural notion of volume, on the space of all paths, because this space is infinite-dimensional. Such a measure $D \gamma$, however, is assumed in the path integral formalism. This problem is not cured by Bohmian mechanics, and, as far as I can see, the problem will never be cured because it is simply a mathematical fact that there is no Lebesgue measure on infinite-dimensional spaces. (However, many astute proposals have been made as to how path integrals can be defined rigorously if one does not insist that it actually be the integral of the action functional $e^{i S[\gamma] / \hbar}$ over path space $[9]$.) I emphasize, however, that path integrals, even when mathematically not clean, often provide correct results by means of a comparatively simple calculation.

\section{Acknowledgments}

This note was inspired by questions from Dennis Smoot of the University of Illinois, Chicago, USA. I thank Jean Bricmont of Université catholique de Louvain, Belgium, for his helpful comments. This work was supported by INFN. 


\section{References}

[1] D. Bohm. A Suggested Interpretation of the Quantum Theory in Terms of "Hidden" Variables, I and II. Phys. Rev., 85:166-193, 1952.

[2] P. R. Holland. The Quantum Theory of Motion. Cambridge University Press, 1993.

[3] K. Berndl, M. Daumer, D. Dürr, S. Goldstein, and N. Zanghì. A survey of Bohmian mechanics. Il Nuovo Cimento, 110B:737-750, 1995.

[4] S. Goldstein. Bohmian mechanics. 2001. In Stanford Encyclopedia of Philosophy. Ed. by E. N. Zalta, published online by Stanford University. http://plato.stanford.edu/entries/qm-bohm/

[5] R. Tumulka. Understanding Bohmian mechanics: A dialogue. Am. J. Phys., 72(9):1220-1226, 2004.

[6] R. P. Feynman. Space-time approach to non-relativistic quantum mechanics. Rev. Mod. Phys., 20:367-387, 1948.

[7] R. P. Feynman and A. R. Hibbs. Quantum Mechanics and Path Integrals. McGraw-Hill, New York, 1965.

[8] L. S. Schulman. Techniques and Applications of Path Integration. John Wiley \& sons, New York, 1981.

[9] S. A. Albeverio and R. J. Høegh-Krohn. Mathematical theory of Feynman path integrals. Lecture Notes in Mathematics, Vol. 523. Springer-Verlag, Berlin-New York, 1976. 